\title{
Could fluorescence-guided surgery be an efficient and sustainable option? A SICE (Italian Society of Endoscopic Surgery) health technology assessment summary
}

\author{
N. Vettoretto ${ }^{1}$ - E. Foglia ${ }^{2}$. L. Ferrario ${ }^{2} \cdot$ C. Gerardi ${ }^{3}$ B. Molteni ${ }^{4} \cdot$ U. Nocco ${ }^{5} \cdot$ E. Lettieri ${ }^{6} \cdot$ S. Molfino ${ }^{4}$ - G. L. Baiocchi ${ }^{4}$.

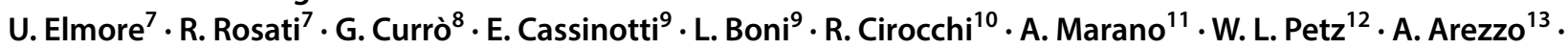 \\ M. A. Bonino ${ }^{13} \cdot$ F. Davini ${ }^{14} \cdot$ A. Biondi ${ }^{15} \cdot$ G. Anania ${ }^{16} \cdot$ F. Agresta $^{17} \cdot$ G. Silecchia ${ }^{18}$
}

Published online: 9 April 2020

(c) Springer Science+Business Media, LLC, part of Springer Nature 2020

\begin{abstract}
Background Indocyanine green fluorescence vision is an upcoming technology in surgery. It can be used in three ways: angiographic and biliary tree visualization and lymphatic spreading studies. The present paper shows the most outstanding results from an health technology assessment study design, conducted on fluorescence-guided compared with standard vision surgery.

Methods A health technology assessment approach was implemented to investigate the economic, social, ethical, and organizational implications related to the adoption of the innovative fluorescence-guided view, with a focus on minimally invasive approach. With the support of a multidisciplinary team, qualitative and quantitative data were collected, by means of literature evidence, validated questionnaires and self-reported interviews, considering the dimensions resulting from the EUnetHTA Core Model.

Results From a systematic search of literature, we retrieved the following studies: 6 on hepatic, 1 on pancreatic, 4 on biliary, 2 on bariatric, 4 on endocrine, 2 on thoracic, 11 on colorectal, 7 on urology, 11 on gynecology, 2 on gastric surgery. Fluorescence guide has shown advantages on the length of hospitalization particularly in colorectal surgery, with a reduction of the rate of leakages and re-do anastomoses, in spite of a slight increase in operating time, and is confirmed to be a safe, efficacious, and sustainable vision technology. Clinical applications are still presenting a low evidence in the literature. Conclusion The present paper, under the patronage of Italian Society of Endoscopic Surgery, based on an HTA approach, sustains the use of fluorescence-guided vision in minimally invasive surgery, in the fields of general, gynecologic, urologic, and thoracic surgery, as an efficient and economically sustainable technology.
\end{abstract}

Keywords Indocyanine green $\cdot$ Surgery $\cdot$ Fluorescence $\cdot$ Laparoscopy

In the last few years, high definition systems (such as $4 \mathrm{~K}$, $3 \mathrm{D}$ vision, and robotic approaches) have raised the surgeon's performance, and patient's safety, potentially decreasing the risk of human errors [1] and biases. Recently image-guided by indocyanine green (ICG) fluorescence has been introduced in minimally invasive clinical practice [2-5]. New

Electronic supplementary material The online version of this article (https://doi.org/10.1007/s00464-020-07542-3) contains supplementary material, which is available to authorized users.

N. Vettoretto

nereovet@gmail.com

Extended author information available on the last page of the article applications such as angiography, sentinel node-guided surgery, and biliary tree visualization have gained wide popularity [6-10].

The fluorescence approach is detected thanks to special cameras that are sensitive to the near-infrared (NIR) spectrum. ICG absorbs NIR light at wavelengths of 800 to $810 \mathrm{~nm}$. This fluorophore emits fluorescence at $830 \mathrm{~nm}$ when bound to tissue proteins if excited, with specific wavelength light in the NIR spectrum $(w 820 \mathrm{~nm})$ or with a laser beam.

Related advantages and drawbacks need to be properly addressed by a multi-dimensional approach, aimed at assessing economic, ethical, social, legal, and organizational dimensions, as required in a full and complete health 
technology assessment (HTA) analysis [11]. This evaluation could help physicians, surgeons, health providers, and payers, in the decision-making process of technologies acquisition, by combining quantitative and qualitative information. Evidence-based results of multiple systematic reviews in various surgical applications of the technology (general, urological, gynecological, thoracic, and cardiac surgery), should be integrated with other relevant aspects of evaluation, thus also comprehending social, organizational, sustainability, affordability, and cost-effectiveness dimensions [12-14].

The SICE Society (Italian Society of Endoscopic Surgery and new technologies, affiliated to the European Society of Endoscopic Surgery-EAES), understanding the factors influencing the use of this technology and the Italian market of reference, tried to investigate the fluorescence dissemination in the Italian healthcare system, by performing a survey among its affiliates [15]. Fifty-six surgeons working in both teaching and community, public and private hospitals (covering $75 \%$ of the Italian regions), answered a questionnaire. $66.1 \%$ of the responders already used fluorescence during their everyday practice. $63.3 \%$ of the surgeons consider ICG fluorescence-guided surgery as an improvement of their practice, while $62.5 \%$ think that this technology can help in surgical education. When asked about the potential growth of ICG, $85 \%$ of those interviewed believe that it has the potential to become a standard vision technology, in the near future.

Despite the potential benefits in using ICG, as well as the clinical evidence produced, no information exists in the Italian setting, with regard to the new technology feasibility in clinical practice. The coverage of the above-mentioned unmet need, could be useful for regional and national governments, who only evaluate and consider the innovations that are able to practically demonstrate their value for money. The study aim was to investigate the effects of vision enhanced by ICG fluorescence compared with standard vision, in different clinical settings: hepatic surgery, upperGI surgery, bariatric surgery, endocrine surgery, thoracic surgery, breast surgery, colorectal surgery, urology, and gynecology.

\section{Methods}

In 2018, a Health Technology Assessment was conducted, by means of the EUnetHTA Core Model [16], comparing the standard vision and the introduction of ICG fluorescence.

Starting from the results of the survey performed by SICE society [15], a multidisciplinary team was created, for the assessment conduction, including the following factors: the major Italian surgical societies, healthcare economists, managerial engineers, HTA and methodology experts, statisticians, and clinical engineers.
Due to the multi-dimensional nature of HTA, the following dimensions were deployed: (i) General relevance of the health problem, useful to analyze the setting in which the new technology could influence the outcome. (ii) Technical relevance, comparing the characteristics of the different fluorescence systems commercialized in Italy, analyzing similar laparoscopic columns, with standard white light. (iii) Safety issues, such as morbidity, mortality, operating time, re-operations, and re-admission. (iv) Efficacy measures derived from the literature review. (v) Economic impact, to define the economic sustainability of the innovative technology. (vi) Equity and accessibility issues, to investigate the accessibility to care. (vii) Ethical and social impact, thus examining the patients' point of view. (viii) Legal issues. (ix) Organizational factors (hospital stay, support for the surgeons, operating time, and learning curve).

For the assessment of the above HTA dimensions, information was retrieved, using different methodological approaches. (i) A structured literature review was used to assess the safety and efficacy profiles, of the technologies under evaluation. (ii) Health economics tools, in terms of economic evaluation of the clinical pathways of the patients, cost-effectiveness and budget impact analyses, considering a 12-month time period from the introduction of the new technology. (iii) Qualitative validated questionnaires, useful for the examination of equity, legal, social, ethical, and organizational aspects, that were completed by a panel of clinicians with expertise in the use of both ICG and the standard vision.

\section{Structured literature review}

Systematic reviews concerning the different clinical settings were conducted to detect evidence in the literature concerning the use of indocyanine in surgery (hepatic surgery, upper-gastrointestinal surgery, bariatric surgery, endocrine surgery, thoracic surgery, colorectal surgery, urology, and gynecology) using the PICO strategy and according to the Cochrane method. Principal scientific databases were searched: PubMed, Embase, Cochrane Library, clinical study registry—clinicaltrial.gov. Titles, abstracts, and full texts, were screened by at least two independent reviewers: one or two surgeons and one methodologist, in addition, potential doubts were solved by a consensus. The screening process was reported using the PRISMA flow-chart [17]. The risk of bias in randomized controlled trials was assessed with a Cochrane tool [18] and the New Castle Ottawa Scale was adopted for observational studies [19].

\section{Health economics tools}

For the deployment of the economic dimension, three different approaches were applied. Firstly, to assess the costs 
of this technology an activity-based costing analysis (ABC) [20-22] was implemented to measure, record, and calculate both the cost and the performance of activities. to produce a real-life economic evaluation, as data from the literature were incomplete, an ICG fluorescence imaging ideal situation was simulated with a pilot study, considering information derived from the survey administered to the centers. Retrospective data during one month of routine ICG fluorescence imaging use were collected from a hospital in which the new technology was routinely employed and compared with the data of the literature concerning standard white light use. In particular, the economic evaluation considered two different phases: [1] the "surgical pathway" including all surgery and technology costs (considering what is consumed "between the walls of the operating theater"); and (2) the "medical pathway" concerning length of stay, laboratory analysis', and other diagnostic procedure costs.

The economic evaluation of the patient's pathway was then integrated, with cost-effectiveness and budget impact analyses. The cost-effectiveness evaluation was developed, to define the technology presenting a better trade-off between the efficacy achieved and the costs absorbed. Secondly, a budget impact analysis (BIA) was conducted, to estimate the financial effects of both the use and the consequent spread of a new healthcare technology in a setting with limited resources [23]. The BIA is a valid support for decision makers and policy makers, as a basis for the evidencebased decision-making process.

The BIA of the present study considered the target population eligible to use ICG, over a one-year time period, in a medium-sized healthcare hospital, during 2018, thus estimating the healthcare expenditure up to 12 months. In particular, the AS IS Scenario (use of only the standard image) was compared with two innovative TO BE scenarios, according to the different market shares devoted to the ICG use, in the different surgical specialties (Table 1), based on experts' opinions.

\section{Qualitative questionnaire}

A specific qualitative questionnaire was created to gather clinicians' perceptions, regarding their ICG use. In particular, the following items related to the specific HTA dimensions were assessed, considering a 7-item Likert scale, ranging from -3 (worst negative impact) to +3 (best positive impact) [24], completed by 17 surgeons using both technologies, referring to 17 Italian Hospitals, thus giving a significant picture of the Italian national landscape.

The safety dimension was investigated by means of the following items: (a) Perception of serious adverse events related to ICG technology. (b) Perception of moderate and mild adverse events. (c) Procedure invasiveness. (d) General safety of the technology. (e) Patient tolerability. (f) Impact of the technology on patient safety. (g) Impact of the technology on clinician safety. (h) Impact of the technology on its repeated use. (i) Impact of the technology on environmental health. (j) Impact of the technology on surgical complications. (k) Safety of ICG injection. (l) Impact of the technology on postoperative pain. (m) Impact of the technology on postoperative infection. (n) Impact of the technology on blood loss.

The efficacy dimension was investigated considering the following aspects: (a) Impact on patients' reported outcome. (b) Impact on the detection rate. (c) Impact on the image quality. (d) Impact on the visualization of vascularization. (e) Impact on the precision of the surgical technique. (f) Impact on the identification of small blood vessels. (g) Impact on the separation/discrimination between healthy and not healthy tissues.
Table 1 Market shares, used in the BIA development (data source: expert opinion)

\begin{tabular}{|c|c|c|c|c|c|c|}
\hline \multirow[t]{2}{*}{ Surgical specialty } & \multicolumn{2}{|c|}{ AS IS scenario } & \multicolumn{2}{|c|}{ TO BE real-life scenario } & \multicolumn{2}{|c|}{$\begin{array}{l}\text { TO BE best case } \\
\text { scenario }\end{array}$} \\
\hline & $\%$ NO ICG & $\%$ ICG & $\%$ NO ICG & $\%$ ICG & $\%$ NO ICG & $\% \mathrm{ICG}$ \\
\hline Hepatic surgery & 100 & 0 & 60 & 40 & 0 & 100 \\
\hline Pancreatic surgery & 100 & 0 & 53 & 47 & 0 & 100 \\
\hline Gastroesophageal surgery & 100 & 0 & 37 & 63 & 0 & 100 \\
\hline $\begin{array}{l}\text { Cholecystectomy and biliary } \\
\text { tree surgery }\end{array}$ & 100 & 0 & 81 & 19 & 0 & 100 \\
\hline Bariatric surgery & 100 & 0 & 88 & 13 & 0 & 100 \\
\hline Endocrine surgery & 100 & 0 & 53 & 47 & 0 & 100 \\
\hline Thoracic surgery & 100 & 0 & 77 & 23 & 0 & 100 \\
\hline Breast surgery & 100 & 0 & 53 & 47 & 0 & 100 \\
\hline Colorectal surgery & 100 & 0 & 21 & 79 & 0 & 100 \\
\hline Urology & 100 & 0 & 53 & 47 & 0 & 100 \\
\hline Gynecology & 100 & 0 & 53 & 47 & 0 & 100 \\
\hline
\end{tabular}


The equity dimension was investigated considering the dimensions listed in the following: (a) Access to care on a local level. (b) Access to care for persons of a legally protected status. (c) Impact on the hospital waiting list. (d) Generation of health migration. (e) Existence of factors limiting the use of the technology for a group of patients. (f) Protection of persons of a legally protected status. (g) General equity. (h) Impact on the access to care, given an optimization of the patients' pathway. (i) Impact on the access to care, given a reduction of surgical re-interventions. (j) Impact on the access to care, given the optimization of the operating time.

The social and ethical dimension comprised the evaluation of the following items: (a) Ability of the technology to protect patients' autonomy. (b) Ability of the technology to protect human rights. (c) Ability of the technology to protect patients' integrity. (d) Ability of the technology to protect patients' dignity. (e) The use of the technology guarantees patients' social values and their willingness to pay. (f) Ability of the technology to protect patients' religion. (g) Impact of the procedure on social costs. (h) Patients and citizens have a good level of understanding of the technology. (i) Impact of the technology on patients' satisfaction. (j) Impact of the technology on patients' perceived quality of life. (k) Impact of the technology on the caregiver's life and perception. (l) Impact of the technology on the patients' recovery. (m) Impact of the technology on the patients' length of stay. (n) Impact of the technology on the follow-up procedures. (o) Impact of the technology on pain management.

The legal dimension was investigated according to these items: (a) Permission level of technology. (b) Need for inclusion of the technology in a registry. (c) Fulfilment of the safety requirements. (d) Production warranties. (e) Price control. (f) The need to regulate the acquisition of the technology. (g) The legislation regulates the technology for all categories of patients. (h) The manual for use of the technology is exhaustive and complete. (i) Infringement of intellectual property rights.

The organizational dimension was analyzed considering the following aspects: (a) The use of the technology requires additional surgeons. (b) The use of the technology requires additional healthcare professionals. (c) The use of the technology requires training course for surgeons. (d) The use of the technology requires training course for healthcare professionals. (e) Hospital meetings. (f) Learning curve for technology use. (g) Equipment update related to technology use. (h) Impact of the technology on workflow standardization. (i) Impact of the technology on the surgeons' productivity. (j) Impact of the technology on the resilience to change. (k) Impact of the technology on change management. (l) Impact of the technology on the optimization of the length of stay. (m) Impact of the technology on operating time. (n) Impact of the technology on the development of fewer adverse events, with a positive impact on the organization. (o) Impact of the technology on the reduction of surgical re-interventions. (p) Impact of the technology on the reduction of follow-up accesses. (q) Impact of the technology to support the surgeon during the surgery. (r) Impact of the technology on purchasing processes. (s) Impact of the technology on internal processes. (t) Impact of the technology on connection processes. (x) Impact of the technology on the patient clinical pathway.

An institutional review board (IRB) approval was not required for this paper.

\section{Results}

\section{Comparison of the technologies under assessment}

Four different systems were introduced in the Italian market from 2013 to 2018. All camera systems have similar resolution characteristics: full HD, camera degree $0-30^{\circ}$, and 3 sensors. There is no market monopoly concerning this technology. All camera systems can be used with white light and near-infrared light. An ECRI Institute (formerly the Emergency Care Research Institute) evaluation was performed on 3 out of 4 systems according to the 5 ECRI criteria: performance, safety, workflow, interoperability, and maintenance. The technologies were comparable and similar in terms of the main characteristics (which neither did not include the surgeons' preference for a certain type of NIR color, nor the possibility to overlap white light images with NIR ones).

\section{Efficacy, safety and organizational results: evidence from the literature review}

Papers selected in each setting varied from 1 to 11 after duplication removal and exclusion of non-pertinent articles, as reported in PRISMA flow diagrams (Online Appendix 1).

The following clinical settings were inserted in PRISMA charts:

- Hepatic surgery: 1337 screened papers, 6 papers included, 1 RCT, 4 case-control study, 1 observational.

- Pancreatic surgery: 1116 screened papers, 1 paper included

- Cholecystectomy and biliary tree: 697 screened paper, 4 papers included, 1 RCT, 2 prospective, 1 retrospective.

- Bariatric surgery: 69 screened papers, 2 papers included case series.

- Endocrine surgery: 1662 screened papers, 4 papers included case control.

- Thoracic surgery: 1205 screened papers, 2 papers included case control. 
- Colorectal surgery: 667 screened papers, 11 papers included, 1 RCT, 10 comparative studies prospective or retrospective.

- Urology: 621 screened papers, 7 papers included case series e match pair analyses.

- Gynecology: 3735 screened papers, 11 paper included, 8 retrospective and 3 prospective.

- Gastric surgery: 1669 screened papers, 2 paper included 1 retrospective and 1 prospective.

- Esophageal surgery: 542 screened papers, 4 paper included 3 retrospective and 1 prospective.

With regard to the evaluation of all the above-mentioned records, the assessment of risk of bias with the Cochrane tool revealed major of trial evaluated with unclear risk of bias for the main domains. On the other hand, the New Castle Ottawa Scale was developed to assess the risk of bias quality in non-randomized studies. Its design is directed at incorporating a bias quality assessment in the interpretation of meta-analytic results of systematic review and metaanalysis. As a result, the risk of bias is not high: the control group was chosen in an interesting manner and the outcomes measurement proved to be relevant in most cases, and both positive and negative outcomes were determined.

\section{General relevance of the pathology}

For the definition of the target population that could benefit from the innovative ICG technology, we considered a potential patient's flow undergoing surgery with the indication to use fluorescence-guided imaging over one year of surgical case-mix taken from a recent (2016) survey performed in Campania Region (Italy) [25].

A total of 23,152 procedures performed by a medium-size hospital on annual basis were considered, excluding 14,586 orthopedic surgeries. Out of the 8566 procedures, 3288 (38\%) were selected to be appropriate for use in our research setting, because of their eligibility to ICG, and because of the presence of literature evidence on the topic, thus being considered the "target population", that could benefit from ICG fluorescence use, considering a 12-month time horizon.

\section{Evaluation of outcomes}

The use of ICG can be different, and we focused our attention on the principal clinical applications of the technology in surgery. Particularly, we studied the impact of the new technology introduction in minimally invasive surgery, with regards to "angiographic" use (visualization of the vascularization of the viscera), "lymphographic" use (visualization of the lymphatic flow or basin after the marking of the lesion, particularly in oncologic surgery), and "biliary tree visualization" (due to the biliary excretion of the fluorescent media).

\section{Hepatic surgery}

The use of ICG in liver surgery may be useful to reduce bile leakage (improve perioperative outcome and hospital stay), to identify additional injuries in association with conventional techniques and to guide the surgeon in obtaining adequate margins during liver resection with consequent lower risk of recurrence and better long-term survival [26-31].

Despite the advantages, the currently available studies do not allow us to support these results with high levels of evidence. More studies are necessary to demonstrate the possible efficacy.

\section{Pancreatic surgery}

This setting lacks evidence, just 1 case series found that the innovative technology is related to a lower development of postoperative pancreatic fistula and to a reduction in operating times [32].

\section{Cholecystectomy}

The use of ICG in gallbladder and biliary surgery can be considered a safe and sustainable technique. Fluorescenceguided surgery can help in identifying extra hepatic biliary structures faster and more frequently when compared to white light [33-35]. It can also help in the recognition of anatomical variants, reducing the risk of bile duct lesions. It could reduce the misinterpretation of normal anatomy and any anatomical variants without interrupting the workflow. Finally, technological innovation can be useful during the learning curve phases, especially for young surgeons [35].

\section{Bariatric surgery}

The use of ICG in bariatric surgery may help in the study of the vascularization of the gastric tubule to reduce leaks. The absence of comparative studies does not allow us to evaluate the technique except in its feasibility $[36,37]$.

\section{Endocrine surgery}

The application of ICG fluorescence in this setting has been studied in the following fields: thyroid surgery with an intravenous injection for visualization and preservation of parathyroid glands; thyroid surgery with an intra-tissue injection for the identification of central neck lymph nodes; adrenal surgery to identify the gland. The evidence is still limited; most studies describe the feasibility of the technique, with partial results [38-40]. Comparative studies are needed. 


\section{Thoracic surgery}

In this setting, fluorescence-guided surgery can be applied to the study of pulmonary vascularization, for the guidance of segmental resections. The outcomes in terms of operative time and bleeding were not significantly different, but all papers evidenced the feasibility of the technique, even if protocols for injection and timing are not yet standardized [41-43].

\section{Colorectal surgery}

In colorectal surgery, two different groups were analyzed, one for the study of vascularization, and one for "nodal navigation", in oncologic lymphadenectomy, without finding any relevant literature for the latter issue [44-52]. In the former setting, a meta-analysis was performed. No statistically significant differences emerged in terms of operative time, re-do anastomosis (10\% after ICG), postoperative hospitalization, overall complications, nor leakage rate. On the other hand, there is a significant difference in the subgroup of rectal resections, in which the ICG significantly reduced the number of leakages (anastomotic re-do surgery by $17 \%$ ) [53-62].

\section{Urology}

A lack of evidence also emerged within this setting. The main purpose of the reports found in the literature was to identify and validate the ICG dosage for use in partial nephrectomy (nephron sparing), and the evaluation of sentinel node in prostatic carcinoma. New possible uses of ICG are in minimally invasive surgery for prostate, bladder, and kidney cancer [63-69].

\section{Gynecology}

This setting mainly concerns the lymphographic use in gynecologic oncology. There are no significant differences in overall detection rate, sensitivity, and predictive value, between ICG and Tc 99-[8 studies] in lymph node detection for ovarian and uterine cancer. A significant superiority of ICG is reported when compared to the radioactive tracer Tc 99 in bilateral lymph node mapping in 4 studies. ICG was significantly superior to blue dye in lymph node mapping
[5 studies]; no allergic complications nor reactions related to this technique are reported; the analyzed studies do not report long-term mortality or follow-up data. More studies are needed to validate this technique [70-80].

\section{Gastric surgery}

At present, there is no evidence to support the use of the ICG for lymphectomy during gastrectomy, even if case reports have tested its use to determine the nodal basin in D2 gastrectomy $[81,82]$.

\section{Esophageal surgery}

The use of the ICG to evaluate the vascularization of the anastomosed gastric tube in the thorax demonstrates a significant reduction in anastomotic fistulas, although there is a low level of evidence. In fact, the procedure is not yet standardized [83-86].

The 11 surgical specialties were divided in two different settings: the use of ICG for vascularization (bariatric surgery, colorectal surgery, esophageal surgery, pancreatic surgery, pulmonary surgery) and the use of ICG for visualization of structures and lymph nodes (gallbladder surgery, endocrine surgery, liver surgery, stomach surgery, gynecology, and urology). Thus, Table 2 depicts the two different efficacy indicators.

\section{Economic and organizational dimensions}

Data available, in terms of evidence in the literature, are still not homogeneous. ICG fluorescence-guided surgery is at the beginning of its dissemination. Therefore, no statistical significance can be found in most of the settings; moreover, there are different results depending on the outcomes under investigation, i.e., "vascularization" or "lymph node study".

In colorectal, particularly in rectal surgery, stronger evidence (confirmed by the experts' opinion and by real world practice) supports a benefit in the use of ICG, with a significant reduction of complications, which could be translated into an optimization of hospitalization time. The final evaluation may depend on overcoming the phase of technological introduction, thus defining potential advantages and greater practicality of use.

\begin{tabular}{llll}
\hline & NO ICG $(\%)$ & ICG $(\%)$ & $p$-value \\
\hline $\begin{array}{l}\text { Vascularization } \\
\text { \% Surgery without anastomoses redo [47, 59] }\end{array}$ & 85.91 & 96.90 & 0.04 \\
$\begin{array}{l}\text { Lymphatic drainage visualization } \\
\% \text { Marked structured and lymph nodes [30] }\end{array}$ & 13.00 & 25.00 & 0.04 \\
\hline
\end{tabular}


Table 3 Patients' pathway average costs

\begin{tabular}{llllc}
\hline & $\begin{array}{l}\text { Cost per patient } \\
\text { NO ICG } \\
\text { AS IS scenario }\end{array}$ & $\begin{array}{l}\text { Cost per patient ICG } \\
\text { TO BE scenario }\end{array}$ & Difference & Difference\% \\
\hline $\begin{array}{l}\text { Technology cost } \\
\begin{array}{l}\text { Surgery cost (without } \\
\text { considering human } \\
\text { resources' cost) }\end{array}\end{array}$ & $€ 4.40$ & $€ 4.72$ & $€ 0.32$ & 7.19 \\
$\begin{array}{l}\text { Human resources costs, } \\
\text { considering the oper- } \\
\text { ating time }\end{array}$ & $€ 224.30$ & $€ 237.39$ & $€ 0.00$ & 0.00 \\
$\begin{array}{l}\text { Medical cost } \\
\text { Average total cost }\end{array}$ & $€ 3201.89$ & $€ 2139.85$ & $€ 13.09$ & \\
\hline
\end{tabular}

Table 4 Average economic value of the patient's pathway, stratified by surgical specialty

\begin{tabular}{lll}
\hline Surgical specialty & $\begin{array}{l}\text { Cost per patient } \\
\text { NO ICG } \\
\text { AS IS scenario }\end{array}$ & $\begin{array}{l}\text { Cost per patient ICG } \\
\text { TO BE scenario }\end{array}$ \\
\hline Hepatic surgery & $€ 10,804.92$ & $€ 9185.30$ \\
Pancreatic surgery & $€ 10,462.25$ & $€ 7743.50$ \\
Gastroesophageal surgery & $€ 13,623.35$ & $€ 10,828.77$ \\
Cholecystectomy and biliary & $€ 5318.00$ & $€ 5319.44$ \\
tree surgery & & \\
Bariatric surgery & $€ 8297.31$ & $€ 7627.01$ \\
Endocrine surgery & $€ 5474.89$ & $€ 5478.46$ \\
Thoracic surgery & $€ 8813.29$ & $€ 7911.60$ \\
Breast surgery & $€ 5191.04$ & $€ 5192.72$ \\
Colorectal surgery & $€ 10,688.68$ & $€ 9,000.60$ \\
Urology & $€ 4732.04$ & $€ 3,835.27$ \\
Gynecology & $€ 4554.10$ & $€ 4,556.85$ \\
\hline
\end{tabular}

Considering the costs of the two different process phases (surgical pathway and medical pathway), on one hand, the examination of the operating times reported a slight disadvantage for ICG $(+7.72 \%)$, thus increasing the 'surgical pathway' costs for surgical interventions in which ICG was used for vascularization. On the other hand, the examination of the "length of stay" has shown an advantage for ICG technology, which can reduce by $42.86 \%$ the hospitalization of patients following a surgical procedure, in which ICG is performed for vascularization.

Table 3 depicts the average comparative economic evaluation of the patients' pathway, thus reporting potential benefits equal to $12.82 \%$, considered as a conservative evaluation, balancing positive and negative factors influencing economic resources absorption.

The economic evaluation of the patient's pathway was then stratified per surgical specialty (Table 4).

Firstly, the cost-effectiveness value (CEV) was defined (Table 5), thus revealing the dominant nature of the innovative ICG technology (the lower the CEV, the preferable the technology).

Results from the BIA (Table 6), show that, from a regional perspective, the ICG introduction, would generate an economic saving, ranging from a minimum of $4 \%$ to a maximum of $8 \%$, strictly dependent on ICG use in hospitals, in the routine surgical routine practice, for conducting 3,288 surgical procedures on an annual basis.

The above mentioned advantages would not only be restricted to the economic sphere, since ICG could also have an impact on the patients' length of stay, and consequently on the induced quantitative organizational impact. It emerged that hospitals could benefit from an organizational advantage of around 12\%, representing 1856
Table 5 Cost-effectiveness analysis

\begin{tabular}{lll}
\hline & $\begin{array}{l}\text { NO ICG } \\
\text { AS IS scenario }\end{array}$ & $\begin{array}{l}\text { ICG } \\
\text { TO BE scenario }\end{array}$ \\
\hline Setting: Vascularization & & \\
Average cost & $€ 9076.61$ & $€ 7665.60$ \\
Efficacy [47, 59]—\% of surgery without re-do anastomosis & $85.91 \%$ & $96.90 \%$ \\
CEV & $10,565.37$ & 7910.44 \\
Setting: Visualization of lymphatic structure & & \\
Average cost & $€ 6482.24$ & $€ 5868.59$ \\
Efficacy [30]—lymphatic structure visualization & $13.00 \%$ & $25.00 \%$ \\
CEV & $49,863.37$ & $23,474.34$ \\
\hline
\end{tabular}


Table 6 Budget impact analysis

\begin{tabular}{|c|c|c|c|c|}
\hline Surgical specialty & AS IS scenario & TO BE real-life scenario & Difference & Difference $\%$ \\
\hline Hepatic surgery & $€ 56,178$ & $€ 52,810$ & $-€ 3368$ & -6 \\
\hline Pancreatic surgery & $€ 790,559$ & $€ 693,686$ & $-€ 96,872$ & -12 \\
\hline Gastroesophageal surgery & $€ 947,571$ & $€ 824,656$ & $-€ 122,915$ & -13 \\
\hline Cholecystectomy and biliary tree surgery & $€ 3,845,782$ & $€ 3,845,975$ & $€ 193$ & 0 \\
\hline Bariatric surgery & $€ 1,133,147$ & $€ 1,121,704$ & $-€ 11,443$ & -1 \\
\hline Endocrine surgery & $€ 1,595,335$ & $€ 1,595,825$ & $€ 491$ & 0 \\
\hline Thoracic surgery & $€ 3,417,365$ & $€ 3,337,903$ & $-€ 79,462$ & -2 \\
\hline Breast surgery & $€ 3,215,375$ & $€ 3,215,866$ & $€ 491$ & 0 \\
\hline Colorectal surgery & $€ 4,865,771$ & $€ 4,260,318$ & $-€ 605,453$ & -12 \\
\hline Urology & $€ 692,718$ & $€ 630,815$ & $-€ 61,904$ & -9 \\
\hline Gynecology & $€ 1,722,712$ & $€ 1,723,202$ & $€ 491$ & 0 \\
\hline Total & $€ 22,282,512$ & $€ 21,302,760$ & $-€ 979,752$ & -4 \\
\hline Hepatic surgery & $€ 56,178$ & $€ 47,757$ & $-€ 8421$ & -15 \\
\hline Pancreatic surgery & $€ 790,559$ & $€ 585,122$ & $-€ 205,436$ & -26 \\
\hline Gastroesophageal surgery & $€ 947,571$ & $€ 753,194$ & $-€ 194,377$ & -21 \\
\hline Cholecystectomy and biliary tree surgery & $€ 3,845,782$ & $€ 3,846,823$ & $€ 1041$ & 0 \\
\hline Bariatric surgery & $€ 1,133,147$ & $€ 1,041,606$ & $-€ 91,541$ & -8 \\
\hline Endocrine surgery & $€ 1,595,335$ & $€ 1,596,375$ & $€ 1041$ & 0 \\
\hline Thoracic surgery & $€ 3,417,365$ & $€ 3,067,732$ & $-€ 349,633$ & -10 \\
\hline Breast surgery & $€ 3,215,375$ & $€ 3,216,416$ & $€ 1041$ & 0 \\
\hline Colorectal surgery & $€ 4,865,771$ & $€ 4,097,312$ & $-€ 768,459$ & -16 \\
\hline Urology & $€ 692,718$ & $€ 561,440$ & $-€ 131,278$ & -19 \\
\hline Gynecology & $€ 1,722,712$ & $€ 1,723,752$ & $€ 1041$ & 0 \\
\hline Total & $€ 22,282,512$ & $€ 20,537,530$ & $-€ 1,744,982$ & -8 \\
\hline
\end{tabular}

hospital days. This advantage could only be achieved in the hypothetical increase of the slot by around $7 \%$ because of the longer operating time needed using ICG (Table 7).
Qualitative impacts: evidence from the professionals' perceptions

As mentioned in the Methods Section, a qualitative questionnaire was administered to 17 surgeons to evaluate their
Table 7 Quantitative organizational impact: release of hospitalization days

\begin{tabular}{lllll}
\hline Surgical specialties & AS IS scenario & $\begin{array}{l}\text { TO BE real-life } \\
\text { scenario }\end{array}$ & Difference & Difference $\%$ \\
\hline Hepatic surgery & 42 & 34 & -7 & -17.14 \\
Pancreatic surgery & 907 & 724 & -183 & -20.21 \\
Upper GI surgery & 858 & 625 & -232 & -27.10 \\
Cholecystectomy and & 2071 & 2071 & 0 & 0.00 \\
$\quad$ biliary tree surgery & & & -22 & -5.36 \\
Bariatric surgery & 410 & 388 & 0 & 0.00 \\
Endocrine surgery & 874 & 874 & -151 & -9.74 \\
Thoracic surgery & 1551 & 1400 & 0 & 0.00 \\
Breast surgery & 1858 & 1858 & $-1,142$ & -33.77 \\
Colorectal surgery & 3382 & 2240 & -118 & -20.21 \\
Urology & 586 & 467 & 0 & 0.00 \\
Gynecology & 2255 & 2255 & $-1,856$ & -12.55 \\
Total & 14,793 & 12,937 & &
\end{tabular}


perceptions on ICG use, focusing on the following dimensions: organizational, equity, ethical, social, and legal (Table 8). It should be noted that the safety and the effectiveness profiles were also assessed in qualitative terms, due to the paucity of evidence-based information derived from scientific literature on the topic.

ICG could be the preferable solution from an effectiveness point of view (average value: 0.54 vs $2.14, p$-value $=0.000$ ). Fluorescence would thus be favorable on patients' reported outcomes, on the detection rate, on image quality, on the visualization of vascularization, on the precision of the surgical technique, on the separation/discrimination between healthy and not healthy tissues. The use of ICG is perceived as improving the precision of the surgical technique, the identification of the blood vessels and the lymph node detection rate, allowing for a better image quality compared with standard white light. Despite no statically differences emerged with regard to the safety aspect perceptions (average value: 0.81 vs $0.98, p$-value $>0.05$ ), ICG is related to a lower occurrence of surgical complications. The only negative aspect is the potential incidence of allergic reaction to ICG, with a consequent impact on its repeated use.

Although ICG is still not commonly used in the Italian setting (Imaging with standard light: 1.19 vs. Imaging with ICG fluorescence: $0.06, p$-value $=0.000$ ), no difference emerged from an equity point of view (Imaging with standard light: 0.75 vs, Imaging with ICG fluorescence: 0.48 , $p$-value $>0.05$ ). It should be noted that ICG presents a potential ability to optimize the patients' pathway: the innovative technology has significant advantages in the optimization of the pathway, especially in terms of better management of adverse events (Imaging with standard light: $0.31 \mathrm{vs.} \mathrm{Imag-}$ ing with ICG fluorescence: $1.50, p$-value $=0.000$ ), as well as the reduction of re-interventions (Imaging with standard light: 0.31 vs. Imaging with ICG fluorescence: 1.88 , $p$-value $=0.000)$, increasing the overall access to care.

Focusing on the patient's point of view (social and ethical impact), the use of ICG would provide a better impact on the patients' quality of life (Imaging with ICG fluorescence: 1.56 vs. Imaging with standard light: 0.56, $p$-value $=0.012$ ) and of the related caregivers (Imaging with ICG fluorescence: 0.88 vs. Imaging with standard light: $0.38, p$-value $=0.035$ ). Furthermore, the innovative technology has a positive impact on the post-intervention recovery time (Imaging with ICG fluorescence: 1.44 vs. Imaging with standard light: $0.63, p$-value $=0.000$ ), on the length of hospitalization (Imaging with ICG fluorescence:1.44 vs. Imaging with standard light: $0.56, p$-value $=0.000$ ) and on the postoperative phase (Imaging with ICG fluorescence: 1.88 vs. Imaging with standard light: $0.81, p$-value $=0.000$ ), compared with conventional white light imaging technique. These results are directly proportional to the image quality of the fluorescence imaging technique and the lower impact of adverse events.

From a legal point of view, the investigated technologies were superimposable in their measurement (Imaging with standard light: 0.70 vs. Imaging with ICG fluorescence: $0.16, p$-value $>0.05$ ). Imaging using ICG is a technology available in clinical practice, but today its utilization is limited to a restricted application field (Limon test and eye fluorangiography). In the investigated settings, ICG is used as an "off-label" indication, with the direct responsibility of the clinician and with the need for an informed consent signed by the patient.

As for the organizational dimension, no statically differences emerged in average terms (Imaging with standard light: 0.20 vs. Imaging with ICG fluorescence: 0.43 , $p$-value $>0.05$ ).

Surgeons agree on the need to train collaborators, both medical managers and staff supporting the procedure. The introduction of fluorescence imaging into clinical practice would allow for an improvement in the organizational process and a consequent optimization of the hospital pathway (Imaging with standard light: 0.31 vs. Imaging with ICG fluorescence: $1, p$-value $=0.029$ ). Finally, surgeons agree in declaring that the new technology allows for a more precise guide of the operator with better results during surgery, given the better image quality of the vision when associated to fluorescence (Imaging with standard light: $0.63 \mathrm{vs.} \mathrm{Imag-}$ ing with ICG fluorescence: $2.75, p$-value $=0.000$ ).

\section{Discussion and conclusion}

The introduction of minimally invasive surgery permits practitioners to improve patient's surgical outcome, reducing the postoperative pain and hospital stay. Research technology tries to investigate how to reduce complications and learning curve, thus improving the benefit for patients [87]. In the last few years, the introduction of fluorescence-guided surgery using ICG seems to be a promising tool to ameliorate surgical outcomes and surgeon performance as well as surgical training. This technique is still under validation, and many clinical trials are ongoing to give more precise answers on the efficacy of this new technology. Unfortunately, the application of fluorescence in most clinical settings needs more trials to test its efficacy in an evidence-based manner.

The Italian Society of Endoscopic Surgery and new technologies (SICE) started to pursue HTA analysis in 2018 with a report on $3 \mathrm{D}$ vs $2 \mathrm{D}$ vision in laparoscopic surgery [1]. Considering the usefulness of that report, we decided to follow the increasing interest of the surgical community in fluorescence-guided surgery as the next step for a full HTA analysis. 
Table 8 Qualitative dimensions: safety, effectiveness, equity, social, legal, and organizational factors

$\begin{array}{ll}\text { Imaging with standard } & \begin{array}{l}\text { Imaging with } \\ \text { light }\end{array} \\ \begin{array}{l}\text { ICG fluores- } \\ \text { cence }\end{array}\end{array}$

\section{Safety profile}

Perception of severe adverse events related to this technology

Perception of moderate and mild adverse events

Procedure invasiveness

$0.94 \quad 0.81$

Safety

$1.75-1.88$

Patient tolerability

$2.25-1.88$

Impact of the technology on patient safety

$1.19 \square \quad 1.56$

Impact of the technology on physician safety

$1.00-1.31$

Impact of the technology on its repeated use

$1.31 \quad 1.06$

Impact of the technology on environmental health

$0.25 \quad 0.31$

Impact of the technology on surgical complications

$0.38 \quad 1.44$

Safety of ICG injection

$0.56 \quad 0.25$

Impact on postoperative pain

$0.19-0.38$

Impact on postoperative infection

$0.13 \quad 0.38$

Impact on blood loss

$0.44 \quad 0.75$

Average value

Effectiveness profile

$\begin{array}{ll}\text { Impact on patients reported outcome } & 0.31\end{array}$

$\begin{array}{lll}\text { Impact on the detection rate } & 0.31 & 2.19\end{array}$

Impact on the image quality $\quad 0.56 \quad 0.38$

Impact on the visualization of vascularization $\quad 0.38 \quad 2.81$

Impact on the precision of the surgical technique $\quad 1.13 \quad 2.44$

Impact on the identification of small blood vessels $\quad 0.44 \quad 2.19$

Impact on the separation/discrimination between healthy and not healthy tissues $\quad 0.63 \quad 2.19$

$\begin{array}{lrl}\text { Average value } & 0.54 & 2.14\end{array}$

Equity profile

Access to care on a local level

1.190 .06

\begin{tabular}{ll} 
Access to care for persons of a legally protected status & 0.88 \\
\hline
\end{tabular}

Impact on the hospital waiting list

$0.19-0.13$

Generation of health migration

$0.31 \quad 1.25$

Existence of factors limiting the use of the technology for a group of patients

$0.13 \quad 0.13$

Protection of persons of a legally protected status

$0.13 \quad 0.19$

General equity of the technology

Impact on the access to care, given an optimization of the patients' pathway

Impact on the access to care, given a reduction of surgical re-interventions

0.31

Impact on the access to care, given the optimization of the operating time

Average value

Social and ethical impact

Ability of the technology to protect patients' autonomy

Ability of the technology to protect human rights

Ability of the technology to protect patients' integrity

Ability of the technology to protect patients' dignity

The use of the technology guarantees patients' social values and their willingness to pay 
Table 8 (continued)

\begin{tabular}{|c|c|c|}
\hline & $\begin{array}{l}\text { Imaging with standard } \\
\text { light }\end{array}$ & $\begin{array}{l}\text { Imaging with } \\
\text { ICG fluores- } \\
\text { cence }\end{array}$ \\
\hline Impact of the technology on the caregiver's life and perception & 0.38 & 0.88 \\
\hline Impact of the technology of the patients' recovery & 0.63 & 1.44 \\
\hline Impact of the technology on the patients' length of stay & 0.56 & 1.44 \\
\hline Impact of the technology on the follow-up procedures & 0.81 & 1.88 \\
\hline Impact of the technology on pain management & 0.56 & 0.63 \\
\hline Average value & 0.49 & 0.87 \\
\hline \multicolumn{3}{|l|}{ Legal impact } \\
\hline Permission level of technology & 0.53 & -0.27 \\
\hline Need for inclusion of the technology in a registry & 0.33 & -0.6 \\
\hline Fulfillment of the safety requirements & 1.4 & 1.27 \\
\hline Production warranties & 1.33 & 1.13 \\
\hline Price control & 0.13 & -0.2 \\
\hline The need to regulate the acquisition of the technology & 0.33 & -0.27 \\
\hline The legislation regulates the technology for all categories of patients & 0.8 & 0.07 \\
\hline The manual for use of the technology is exhaustive and complete & 1.27 & 0.13 \\
\hline Infringement of intellectual property rights & 0.2 & 0.2 \\
\hline Average value & 0.7 & 0.16 \\
\hline \multicolumn{3}{|l|}{ Organizational impact } \\
\hline The use of the technology requires additional surgeons & 0 & -0.13 \\
\hline The use of the technology requires additional healthcare professionals & -0.13 & -0.31 \\
\hline The use of the technology requires training course for surgeons & -0.19 & -1.19 \\
\hline The use of the technology requires training course for healthcare professionals & -0.19 & -0.94 \\
\hline Hospital meetings & -0.06 & -0.88 \\
\hline Learning curve for technology use & 0.31 & -0.06 \\
\hline Equipment update related to technology use & -0.38 & -1 \\
\hline Impact of the technology on workflow standardization & 0.44 & 0.63 \\
\hline Impact of the technology on the surgeons' productivity & 0.5 & 0.56 \\
\hline Impact of the technology on the resilience to change & 0.31 & 0.13 \\
\hline Impact of the technology on change management & 0 & 0 \\
\hline Impact of the technology on the optimization of the length of stay & 0.5 & 1.38 \\
\hline Impact of the technology on the operating time & 0.38 & 0.5 \\
\hline $\begin{array}{l}\text { Impact of the technology on the development of fewer adverse events, with a positive impact on } \\
\text { the organization }\end{array}$ & 0.44 & 1.63 \\
\hline Impact of the technology on the reduction of surgical re-interventions & 0.25 & 1.5 \\
\hline Impact of the technology on the reduction of follow-up accesses & 0.13 & 1.06 \\
\hline Impact of the technology to support the surgeon during the surgery & 0.63 & 2.75 \\
\hline Impact of the technology on purchasing processes & 0.13 & 0.19 \\
\hline Impact of the technology on internal processes & 0.38 & 1.06 \\
\hline Impact of the technology on connection processes & 0.38 & 1.13 \\
\hline Impact of the technology on the patient clinical pathway & 0.31 & 1 \\
\hline Average value & 0.20 & 0.43 \\
\hline
\end{tabular}

Preliminary results reported that the points of strength of this technology mainly concern the dimensions of effectiveness, economic impact, and organizational impact, although the absence of studies with a high level of evidence is currently a lack.
The budget impact analysis foresees a reduction in costs, thus freeing up some economic and organizational resources. From an economic point of view, results suggested the opportunity to achieve significant economic savings, ranging from -4 to $-8 \%$, even in a conservative 
scenario of analysis. This analysis proves that investment in this field could be feasible and sustainable.

The adoption of ICG technology would allow for an optimization of the patients' surgical pathway, and the investment due to the slight increase in the operating time (only $15 \mathrm{~min}$ ), could be compensated by a reduction in the length of the hospitalization. In colorectal surgery, the visualization of anastomotic tissue vitality seems to reduce the number of leakages and re-do anastomoses [54-64], and the same advantages are seen in oesophageal [84-86] surgery. Moreover, this technique might show promising results in surgical navigation and lymphnode mapping in most oncological settings regarding gynecologic [70-80], urologic [63-69], bariatric [36, 37], gastric [81-83], endocrine [38-40], pancreatic [32], thoracic [41-44] and hepatic [26-31] surgery. A better view of the biliary structures in cholecystectomy (especially in acute settings) could be a turning point for the reduction of biliary injuries [33-35]. All these clinical advantages must be proven by new and ongoing trials, but they have to be associated to a sustainability of the costs required for the new technology. It should be noted that the above results have been achieved without taking into consideration the economic evaluation of all the possible procedure-related adverse events, which could generate an incremental improvement in the patients' clinical pathway. This could be a topic for further research, since the reduction of severe or mild complications could positively influence the release of both economic and human resources to be reinvested in the acquisition of the innovative technology. On the other hand, in the real practice, the experts suggested that the new technology learning curve embraces a short-term time horizon. This doesn't classify ICG surgical practice as a disruptive innovation, thus being easily acceptable and valid both for novice and experienced surgeons, differently from other healthcare technologies affected by the digital divide [1]. In addition, the results of this HTA activity could be useful for the creation of an ad-hoc, head-to-head clinical trials, comparing the use of ICG to standard vision in all the clinical settings where evidence is actually lacking, to reinforce the perceptions exposed herein. However, it is hoped that we have collected most of the key publications for giving an overview of the indocyanine green fluorescence technology in surgery, and its most important emerging clinical application.

Despite the aforementioned drawbacks, this paper would encourage surgeons to use ICG whenever available according to the clinical needs, leading to a better vision during surgery, with an improvement in the safety profiles and in the patients' outcomes, offering both challenges and the potential for totally new clinical applications.

\section{Compliance with ethical standards}

Disclosures Doctors Vettoretto Nereo, Foglia Emanuela, Ferrario Lucrezia, Gerardi Chiara, Molteni Beatrice, Nocco Umberto, Lettieri Emanuele, Molfino Sarah, Baiocchi Gianluca, Elmore Ugo, Rosati Riccardo, Currò Giuseppe, Cassinotti Elisa, Boni Luigi, Cirocchi Roberto, Marano Alessandra, Petz Wanda Luisa, Arezzo Alberto, Bonino Marco Augusto, Davini Federico, Biondi Alberto, Anania Gabriele, Agresta Ferdinando, Silecchia Gianfranco have no conflicts of interest or financial ties to disclose.

\section{References}

1. Vettoretto N (2018) Why laparoscopists may opt for three-dimensional view: a summary of the full HTA report on 3D versus 2D laparoscopy by S.I.C.E. (Società Italiana di Chirurgia Endoscopica e Nuove Tecnologie). Surg Endosc 32:2986-2993

2. Kamisaka K, Yatsuji Y, Yamada H, Kameda H (1974) The binding of indocyanine green and other organic anions to serum proteins in liver diseases. Clin Chim Acta 53:255

3. Alander J (2012) A review of indocyanine green fluorescent imaging in surgery. Int $\mathbf{J}$ Biomed Imaging. https://doi. org/10.1155/2012/940585

4. Mishra A (1990s) Cyanine during the 1990s: a review. Chem Rev 100(6): 1973

5. Kang Y (2010) Dynamic fluorescence imaging of indocyanine green for reliable and sensitive diagnosis of peripheral vascular insufficiency. Microvasc Res 80:552

6. Schaafsma B (2011) The clinical use of Indocyanine green as a near-infrared fluorescent contrast agent for image-guided oncologic surgery. J Surg Oncol 104:323

7. Boni L (2015) Clinical applications of indocyanine green (ICG) enhanced fluorescence in laparoscopic surgery. Surg Endosc 29:2046-2055

8. Boni L (2017) Indocyanine green fluorescence angiography during laparoscopic low anterior resection: results of a case-matched study. Surg Endosc 31:1836-1840

9. Molfino S (2018) Different ways to manage indocyanine green fluorescence to different purposes in liver surgery: a systematic review. J Periton. https://doi.org/10.4081/joper.2018.96

10. Molteni B (2018) Gastric cancer indocyanine green lymph node navigation surgery: systematic review. J Periton. https://doi. org/10.4081/joper.2018.97

11. Drummond MF (2008) Key principles for the improved conduct of health technology assessments for resource allocation decisions. Int J Technol Assess Health Care 24(3):244-258

12. Marshall D (2009) Design and analysis issues for economic analysis alongside clinical trials. Med Care 47(7 Suppl 1):S14-S20

13. America IoM(CoQoHCi) (2001) Crossing the quality chasm: a new health system for the 21 st century. National Academies Press, Washington, DC

14. Mc PB (1989) Randomized clinical trials in surgery. Int J Technol Assess Health Care 5:317-332

15. SICE, Società Italiana di Chirurgia Endoscopica e nuove tecnologie (2019) Full-report H.T.A. "FLUO project" Valutazione multidimensionale sull'utilizzo della visione con fluorescenza e verde di indocianina in specifici setting chirurgici, pp 133-137. https ://siceitalia.com/wp-content/uploads/2019/05/2019-05-24-Repor t-FLUO-HTA-Definitivo.pdf. Accessed 6 April 2020

16. Radaelli G (2014) Implementation of EUnetHTA core Model ${ }^{\circledR}$ in Lom-bardia: the VTS framework. Int J Technol Assess Health Care 30:105-112 
17. Preferred Reporting Items for Systematic Reviews and MetaAnalyses. http://prisma-statement.org/. Accessed 6 April 2020

18. Higgins JPT, Green S (eds.) (2011) Cochrane handbook for systematic reviews of interventions version 5.1.0. The cochrane collaboration. http://handbook-5-1.cochrane.org/. Accessed 6 April 2020

19. Wells GA, Shea B, O'Connell D et al. The Newcastle-Ottawa Scale (NOS) for assessing the quality of nonrandomised studies in meta-analyses. http://www.ohri.ca/programs/clinical_epidemiolo gy/oxford.asp. Accessed 6 April 2020

20. Raffish N (1991) Glossary of activity-based management. J Cost Manage 5:53-63

21. Drucker P (1995) The information executives truly need. Harvard Bus Rev 73:54-64

22. Vagoni E (2003) L'activity based costing in sanità: il caso dell'ossigeno-terapia. Mecosan 47:149-161

23. Mauskopf J (2007) Principles of good practice for budget impact analysis: report of the ISPOR Task Force on good research practices-budget impact analysis. Value Health

24. Mitton C (2011) Difficult decisions in times of constraint: criteria based resource allocation in the vancouver coastal health authority. BMC Health Serv Res 11(1):169

25. Campania AASDR (2015) Sorveglianza delle infezioni del sito chirurgico in Campania

26. Kaibori M (2011) Intraoperative indocyanine green fluorescent imaging for prevention of bile leakage after hepatic resection. Surgery 150(1):91-98

27. van der Vorst JR (2013) Near-infrared fluorescence-guided resection of colorectal liver metastases. Cancer 119(18):3411-3418

28. Abo T (2015) Usefulness of intraoperative diagnosis of hepatic tumors located at the liver surface and hepatic segmental visualization using indocyanine green-photodynamic eye imaging. Eur J Surg Oncol 41:257-264

29. Zhang YM (2017) Liver tumor boundaries identified intraoperatively using real-time indocyanine green fluorescence imaging. $\mathrm{J}$ Cancer Res Clin Oncol 143:51-58

30. Handgraaf HJM (2017) Long-term follow-up after near-infrared fluorescence-guided resection of colorectal liver metastases: a retrospective multicenter analysis. Eur J Surg Oncol 43:1463-1471

31. Aoki T (2018) Determination of the surgical margin in laparoscopic liver resections using infrared indocyanine green fluorescence. Langenbeck's Arch Surg 403:671-680

32. Rho SY (2018) Indocyanine green perfusion imaging-guided laparoscopic pancreaticoduodenectomy: potential application in retroperitoneal margin dissection. J Gastrointest Surg 22(8):1470-1474

33. Buchs NC (2013) Real-time near-infrared fluorescent cholangiography could shorten operative time during robotic single-site cholecystectomy. Surg Endosc 27(10):3897-3901

34. van Dam DA (2015) Comparing near-infrared imaging with indocyanine green to conventional imaging during laparoscopic cholecystectomy: a prospective crossover study. J Laparoendosc Adv Surg Tech A 25(6):486-492

35. Sherwinter D (2012) The efficacy of fluorescent cholangiography using the spy scope' system, a randomized control trial. Surg Endosc Other Interv Tech 26:S204

36. Frattini F (2015) Indocyanine green-enhanced fluorescence in laparoscopic sleeve gastrectomy. Obes Surg 25(5):949-950

37. Ortega CB (2018) The use of fluorescence angiography during laparoscopic sleeve gastrectomy. JSLS 22:2

38. Kahramangil B (2017) The use of near-infrared fluorescence imaging in endocrine surgical procedures. J Surg Oncol 115(7):848-855

39. Kahramangil B (2017) Comparison of indocyanine green fluorescence and parathyroid autofluorescence imaging in the identification of parathyroid glands during thyroidectomy. Gland Surg 6(6):644-648
40. Yu HW (2017) Intraoperative localization of the parathyroid glands with indocyanine green and Firefly $(\mathrm{R})$ technology during BABA robotic thyroidectomy. Surg Endosc 31(7):3020-3027

41. Yasuo S (2012) A simple and effective technique for identification of intersegmental planes by infrared thoracoscopy after transbronchial injection of indocyanine green. J Thorac Cardiovasc 143(6):1330-1335

42. Tarumi S (2014) Clinical trial of video-assisted thoracoscopic segmentectomy using infrared thoracoscopy with indocyanine green. Eur J Cardiothorac Surg 46:112-115

43. Digesu CS (2018) Long-term outcomes after near-infrared sentinel lymph node mapping in non-small cell lung cancer. J Thorac Cardiovasc Surg 155(3):1280-1291

44. Bourgeois P Near-infra-red (NIR) fluorescence imaging of « sentinel » lymph nodes in cancers after the intra-venous injection (IV) of free indocyanine green (ICG)

45. Chand M (2018) Feasibility of fluorescence lymph node imaging in colon cancer: FLICC. Tech Coloproctol 22(4):271-277

46. Currie AC (2017) A pilot study to assess near infrared laparoscopy with indocyanine green (ICG) for intraoperative sentinel lymph node mapping in early colon cancer. Eur J Surg Oncol 43(11):2044-2051

47. Tamura K (2018) Using indocyanine green fluorescent imaging to successfully resect metachronous regional lymph node recurrence of rectosigmoid cancer. Asian J Endosc Surg 11(1):47-49

48. Weixler B (2017) Sentinel lymph node mapping with isosulfan blue or indocyanine green in colon cancer shows comparable results and identifies patients with decreased survival: a prospective single-center trial. World J Surg 41(9):2378-2386

49. Andersen HS (2017) In vivo and ex vivo sentinel node mapping does not identify the same lymph nodes in colon cancer. Int J Colorectal Dis 32(7):983-990

50. Liberale G (2016) Sentinel lymph node detection by blue dye versus indocyanine green fluorescence imaging in colon cancer. Anticancer Res 36(9):4853-4858

51. Watanabe $\mathbf{J}$ (2017) Evaluation of lymph flow patterns in splenic flexural colon cancers using laparoscopic real-time indocyanine green fluorescence imaging. Int J Colorectal Dis 32(2):201-207

52. Boni L, Fingerhut A, Marzorati A, Rausei S, Dionigi G, Cassinotti E (2017) Indocyanine green fluorescence angiography during laparoscopic low anterior resection: results of a casematched study. Surg Endosc Interv Tech 31(4):1836-1840

53. Ciarleglio FA, Brolese A, Marcucci S, Valduga P, Beltempo $\mathrm{P}$, Prezzi $\mathrm{C}$ et al Preliminary results on application of da vinci fluorescence imaging vision system with indocyanine green (ICG) in robotic colo rectal surgery. Surgical endoscopy and other interventional techniques Conference: 24th international congress of the European association for endoscopic surgery, EAES 2016 Netherlands. 2017;31(2 Supplement 1):S470.

54. Keller D, Ibarra S, Flores JR, Haas EM (2016) Impact of fluorescence angiography on clinical and financial outcomes in colorectal surgery: a case matched series. Gastroenterology 150(4):S1242

55. Kim JC, Lee JL, Yoon YS, Alotaibi AM, Kim J (2016) Utility of indocyanine-green fluorescent imaging during robot-assisted sphincter-saving surgery on rectal cancer patients. Int J Med Robot Comput Assist Surg 12(4):710-717

56. Quartey B, Chinn B, Wilkins K, Notaro J, Alva S, Saleem A et al (2016) Real time intraoperative assessment of colonic perfusion in colon and rectal surgery. Dis Colon Rectum 59(5):e339-e340

57. Jafari MD, Lee KH, Halabi WJ, Mills SD, Carmichael JC, Stamos MJ et al (2013) The use of indocyanine green fluorescence to assess anastomotic perfusion during robotic assisted laparoscopic rectal surgery. Surg Endosc 27(8):3003-3008

58. Martin-Perez B, Otero-Piñeiro A, DeLacy-Oliver B, Pena-Lopez R, Arroyave MC, Fernandez-Hevia M et al (2017) Transanal total 
mesorectal excision for rectal cancer: assessment with indocyanine green. Surg Endosc Interv Tech 31:S204

59. Mizrahi I, Abu-Gazala M, Rickles A, Fernandez L, Petrucci A, Wolf J et al (2017) Indocyanine green fluorescence angiography for low anterior resection: results of a comparative cohort study. Colorectal Dis 19:37

60. Moore C, Turner J, Naddell C, Okonkwo A, Childs E, Clark C (2016) Short term outcomes in laparoscopic colorectal surgery with and without the use of fluorescent angiography. Surg Endosc Interv Tech 30:S347

61. Ramphal W, Crolla RMPH, Gobardhan PD, Wijsman JH, Van Der Schelling GP, Schreinemakers JMJ (2017) Colorectal perfusion with indocyanine green in colonic resection performed in robotic surgery: reducing the risk of anastomotic leakage? Surg Endosc Interv Tech 31(2):S4

62. Rosati R, De Nardi P, Maggi G, Maggiore R, Elmore U, Boni L et al (2017) Anastomosis perfusion in laparoscopic colorectal resection with indocyanine green angiography: preliminary results of a multicenter RCT. Surg Endosc Interv Tech 31(2):S24

63. KleinJan GH (2014) Optimisation of fluorescence guidance during robot-assisted laparoscopic sentinel node biopsy for prostate cancer. Eur Urol 66(6):991-998

64. Lanchon C et al (2018) Super-selective robot-assisted partial nephrectomy using near-infrared flurorescence versus earlyunclamping of the renal artery: results of a prospective matchedpair analysis. IBJU 44:53-62

65. McClintock TR (2014) Can selective arterial clamping with fluorescence imaging preserve kidney function during robotic partial nephrectomy? Urology 84(2):327-332

66. Borofsky MS (2012) Near-infrared fluorescence imaging to facilitate super-selective arterial clamping during zero-ischaemia robotic partial nephrectomy. BJUI 111:604-610

67. Krane LS (2012) Is near infrared fluorescence imaging using indocyanine green dye useful in robotic partial nephrectomy: a prospective comparative study of 94 patients. Urology 80:110-118

68. Markuszewski M (2015) Comparison of real-time fluorescent indocyanine green and $(99 \mathrm{~m}) \mathrm{Tc}-$ nanocolloid radiotracer navigation in sentinel lymph node biopsy of penile cancer. Clin Genitourin Cancer 13(6):574-580

69. Manny TB (2014) Fluorescence-enhanced robotic radical prostatectomy using real-time lymphangiography and tissue marking with percutaneous injection of unconjugated indocyanine green: the initial clinical experience in 50 patients. Eur Urol 65:1162-1168

70. Tanaka $\mathrm{T}$ (2018) The detection of sentinel lymph nodes in laparoscopic surgery can eliminate systemic lymphadenectomy for patients with early stage endometrial cancer. Int J Clin Oncol 23:305-313

71. Martinelli F (2017) Sentinel node mapping in endometrial cancer following hysteroscopic injection of tracers: a single center evaluation over 200 cases. Gynecol Oncol 146(3):525-530

72. Di Martino G (2017) Indocyanine green versus radiotracer with or without blue dye for sentinel lymph node mapping in stage $\% 3 \mathrm{eIB} 1$ cervical cancer $(\% 3 \mathrm{e} 2 \mathrm{~cm})$. J Minim Invas Gynecol 24(6):954-959

73. Soergel P (2017) Sentinel lymphadenectomy in vulvar cancer using near-infrared fluorescence from indocyanine green compared With technetium 99m nanocolloid. Int J Gynecol Cancer 27(4):805-812

74. Eriksson A (2017) A comparison of the detection of sentinel lymph nodes using indocyanine green and near-infrared fluorescence imaging versus blue dye during robotic surgery in uterine cancer. Int J Gynecol Cancer 27(4):743-747

75. Buda A (2017) A comparison of the detection of sentinel lymph nodes using indocyanine green and near-infrared fluorescence imaging versus blue dye during robotic surgery in uterine cancer. Int J Gynecol Cancer 27(4):743-747

76. Papadia A (2017) Sentinel lymph node mapping in patients with stage I endometrial carcinoma: a focus on bilateral mapping identification by comparing radiotracer Tc $99 \mathrm{~m}$ with blue dye versus indocyanine green fluorescent dye. J Cancer Res Clin Oncol 143(3):475-480

77. Holloway RW (2017) A prospective cohort study comparing colorimetric and fluorescent imaging for sentinel lymph node mapping in endometrial cancer. Ann Surg Oncol 24(7):1972-1979

78. Imboden S (2015) A comparison of radiocolloid and indocyanine green fluorescence imaging, sentinel lymph node mapping in patients with cervical cancer undergoing laparoscopic surgery. Ann Surg Oncol 22(13):4198-4203

79. Tanner EJ (2015) Factors associated with successful bilateral sentinel lymph node mapping in endometrial cancer. Gynecol Oncol 138(3):542-547

80. How J (2015) Comparing indocyanine green, technetium, and blue dye for sentinel lymph node mapping in endometrial cancer. Gynecol Oncol 137(3):436-442

81. Ichikura T (2002) Sentinel node concept in gastric carcinoma. World J Surg 26(3):318-322

82. Lan YT (2017) A pilot study of lymph node mapping with indocyanine green in robotic gastrectomy for gastric cancer. SAGE Open Med 21:5

83. Noma K (2018) Visualized evaluation of blood flow to the gastric conduit and complications in esophageal reconstruction. J Am Coll Surg 226(3):241

84. Karampinis I (2017) Indocyanine green tissue angiography affects anastomotic leakage after esophagectomy. A retrospective, casecontrol study. Int J Surg 48:210-214

85. Campbell C (2015) Conduit vascular evaluation is associated with reduction in anastomotic leak after esophagectomy. J Gastrointest Surg 19(5):806-812

86. Dalton BGA (2018) Near infrared perfusion assessment of gastric conduit during minimally invasive Ivor Lewis esophagectomy. Am J Surg 216(3):524-527

87. Arezzo A (2014) The past, the present, and the future of minimally invasive therapy in laparoscopic surgery: a review and speculative outlook. Minim Invas Ther Allied Technol 23(5):253-260

Publisher's Note Springer Nature remains neutral with regard to jurisdictional claims in published maps and institutional affiliations. 


\section{Affiliations}

\section{N. Vettoretto ${ }^{1}$ - E. Foglia ${ }^{2}$. L. Ferrario ${ }^{2}$. C. Gerardi ${ }^{3}$ B. Molteni ${ }^{4}$ - U. Nocco ${ }^{5}$ - E. Lettieri ${ }^{6}$. S. Molfino ${ }^{4}$ - G. L. Baiocchi ${ }^{4}$.

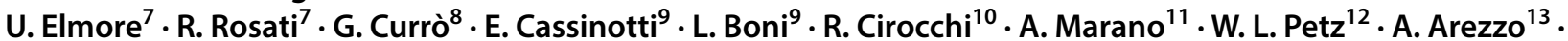

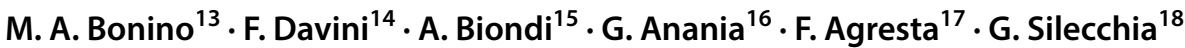

1 Chirurgia Montichiari, Azienda Socio Sanitaria Territoriale Degli Spedali Civili, V.le Ciotti 154, Montichiari, 25018 Brescia, Italy

2 LIUC - Università Cattaneo, Castellanza, VA, Italy

3 Centro di Politiche Regolatorie, Istituto di Ricerche Farmacologiche "Mario Negri” IRCCS, Milan, Italy

4 Department of Clinical and Experimental Surgery, University of Brescia, Brescia, Italy

5 Ingegneria Clinica, Azienda Socio Sanitaria Territoriale dei Sette Laghi, Varese, Italy

6 School of Management, Department of Management, Economics and Industrial Engineering, Politecnico, Milano, Italy

7 Department of Gastrointestinal Surgery, IRCCS San Raffaele Scientific Institute, Vita-Salute San Raffaele University, Milan, Italy

8 Department of Human Pathology of Adult and Evolutive Age, University Hospital of Messina, Messina, Italy

9 Chirurgia Generale, Fondazione IRCCS - Ca' Granda - Ospedale Maggiore Policlinico - University of Milan, Milan, Italy
10 Department of Surgical Sciences, University of Perugia, Perugia, Italy

11 Chirurgia Generale ed Oncologica, Azienda Ospedaliera S. Croce e Carle, Cuneo, Italy

12 Chirurgia, IEO, European Institute of Oncology IRCCS, Milan, Italy

13 Department of Surgical Sciences, University of Torino, Turin, Italy

14 Centro multidisciplinare Chirurgia Robotica, Chirurgia Toracica mini-invasiva e Robotica, Azienda Ospedaliero-Universitaria Pisana, Pisa, Italy

15 Chirurgia Generale, Fondazione Policlinico Universitario A. Gemelli IRCSS, Rome, Italy

16 Chirurgia Generale, University of Ferrara, Ferrara, Italy

17 Chirurgia Generale, Azienda ULSS 5 "Polesana", Hospital of Adria, Adria, RO, Italy

18 Department of Medical-Surgical Sciences and Biotechnologies, Sapienza University of Rome-Polo Pontino, Rome, Italy 